\title{
The Role of RNA and RNA Processing in Neurodegeneration
}

\author{
Jean-Marc Gallo, ${ }^{1}$ Peng Jin, ${ }^{2}$ Charles A. Thornton, ${ }^{3}$ Hong Lin, ${ }^{4}$ Janice Robertson, ${ }^{5}$ Ian D’Souza, ${ }^{6}$ and \\ William W. Schlaepfer ${ }^{4}$ \\ ${ }^{1}$ Medical Research Council Centre for Neurodegeneration Research and Department of Neurology, Institute of Psychiatry, King's College London, London \\ SE5 8AF, United Kingdom, 2Department of Human Genetics, Emory University School of Medicine, Atlanta, Georgia 30322, ${ }^{3}$ Department of Neurology, \\ University of Rochester School of Medicine and Dentistry, Rochester, New York 14642, ${ }^{4}$ Division of Neuropathology, University of Pennsylvania Medical \\ School, Philadelphia, Pennsylvania 19104-6100, ${ }^{5}$ University of Toronto, Centre for Research in Neurodegenerative Diseases, Toronto, Ontario, Canada \\ M5S 3H2, and 'University of Washington and Veterans Affairs Medical Center, Seattle, Washington 98108
}

Key words: RNA; triplet repeat expansions; tau; amyotrophic lateral sclerosis; neurofilaments; alternative splicing

From its transcription from DNA, RNA undergoes a complex processing, including splicing and editing, and associates with specific proteins that determine its subcellular localization and stability. It is now clear that many cellular activities involve some aspects of RNA biology; in neurons, synaptic activity is a typical process in which RNA plays a critical role. For instance, proper targeting of specific mRNAs to dendrites allows rapid local protein translation at postsynaptic sites in response to stimulation by neurotransmitters or neurotrophic factors, an important component of synaptic plasticity. Repression of local translation at the postsynaptic level by noncoding RNAs, including microRNAs, offers another level of regulation mediated by RNA (Kosik and Krichevsky, 2005). Increased complexity of the proteome and functional diversity can be generated from a limited number of genes ( $\sim 20,000-25,000$ in the human genome) by including or skipping specific exons through pre-mRNA alternative splicing. In neurons, alternative splicing generates neurotransmitter receptors with different specificities and coordinates the activity of protein networks at the synapse (Ule et al., 2005). RNA processing participates in cellular surveillance by degrading mRNA containing premature termination signals through nonsensemediated decay, hence avoiding their translation into truncated proteins. Proteins binding to defined RNA elements control RNA metabolism at all levels. Of particular interest are proteins binding to the $5^{\prime}$ - and $3^{\prime}$-untranslated regions (UTRs) of the mRNA that regulate translation and mRNA stability.

Given the importance of RNA processing in governing neuronal function, it will come as no surprise that its impairment can lead to neuronal dysfunction or, in extreme cases, neuronal degeneration. A prime example of this is spinal muscular atrophy (SMA), an autosomal recessive lower motor neuronopathy disorder of childhood. SMA is caused by mutations in the SMN1 gene leading to the loss of its product, the survival of motor neuron (SMN) protein (Frugier et al., 2002). The product of a second copy of the gene, SMN2, cannot compensate the loss of SMN because of a mutation in the last exon that affects its splic-

Received Aug. 16, 2005; revised Sept. 17, 2005; accepted Sept. 17, 2005.

Correspondence should be addressed to Jean-Marc Gallo, Medical Research Council Centre for Neurodegeneration Research and Department of Neurology, Institute of Psychiatry, King's College London, Box P037, De Crespigny Park, London SE5 8AF, UK. E-mail: j.gallo@iop.kcl.ac.uk.

DOI:10.1523/JNEUROSCI.3453-05.2005

Copyright $\odot 2005$ Society for Neuroscience $\quad$ 0270-6474/05/2510372-04\$15.00/0 ing, resulting in predominantly truncated, unstable forms of SMN. Interestingly, SMA is a disease of RNA processing in a second way as the SMN protein is itself an RNA-binding protein involved in various aspects of RNA function such as splicing (Gubitz et al., 2004) and mRNA localization to the axon (Rossoll et al., 2003). New evidence has emerged recently for a widespread involvement of RNA-mediated mechanisms in a number of degenerative pathologies of the CNS. RNA-based mechanisms have been implicated in conditions ranging from triplet repeat expansion diseases, amyotrophic lateral sclerosis (ALS), and dementias, leading to the recognition of new pathogenic pathways in which pathogenicity is initiated at the RNA level, hence challenging the traditional view that pathogenesis is triggered by abnormal protein processing, be it aberrant posttranslational modifications, proteolytic processing, or misfolding. The following examples illustrate two major RNA-dependent mechanisms involved in neurodegeneration: trans-dominant effect of RNA on the activity of RNA-binding proteins and aberrant alternative splicing.

\section{Trans-dominant pathogenic role of RNA: abnormal activity of UTR-binding proteins}

A number of human diseases are caused by triplet repeat expansions in nontranslated regions of the responsible genes (Ranum and Day, 2004). Fragile X syndrome (FXS) is caused by a CGG expansion in the $5^{\prime}$-UTR of the Fmrl gene, consistent with a loss-of-function mechanism in which these expansions prevent the expression of the normal protein. Conversely, the discovery of the dominant mutation in myotonic dystrophy type 1 (DM1) results in a long expansion of 300-12,000 nucleotides of CUG repeats in the $3^{\prime}$-UTR of the mRNA encoding myotonic dystrophy protein kinase did not fit into a loss-of-function category. A second form of myotonic dystrophy, DM type 2 (DM2), also is caused by a very large expansion of a repeat, although the offending RNA sequence in this case is a CCUG tetramer repeat located in an intron.

FXS carriers have Fmr1 alleles, called premutations, with an intermediate number of CGG repeats between patients $(>200$ repeats) and normal individuals ( $<55$ repeats). Some Fmr1 premutation carriers aged in their 50s and older develop a distinct tremor/ataxia syndrome, designated "fragile-X-associated tremor/ataxia syndrome" (FXTAS), and consisting of progressive intention tremor, gait ataxia, parkinsonism, and autonomic dys- 
function (Hagerman and Hagerman, 2004). The most striking neuropathological feature associated with FXTAS is the presence of eosinophilic, intranuclear inclusions in both neurons and astrocytes (Hagerman and Hagerman, 2004). However, no evidence of neurodegeneration has been found in either full mutation patients or in the Fmrl knock-out mouse model, indicating that modulation of the levels of the Fmrl gene product, the translational repressor FMRP (fragile X mental retardation protein), is not likely to account for the neurodegeneration. The fact that Fmr1 premutation alleles differ from both normal and full mutation alleles by producing more Fmrl transcripts with lengthy rCGG repeats raises the possibility that the neurodegeneration is RNA mediated (Tassone et al., 2000). This notion is further supported by the observation of intranuclear inclusions in a "knockin" mouse model in which the five endogenous CGG repeats had been replaced with an $\sim 100$ CGG-repeat fragment within the Fmr1 gene (Willemsen et al., 2003). To directly test the toxicity of rCGG repeats, Jin et al. (2003) have established a Drosophila model that ectopically expresses the human Fmr1 5'-UTR containing either normal- or premutation-length rCGG repeats. These experiments showed that fragile X premutation rCGG repeats alone are sufficient to cause neurodegeneration in a dosageand repeat-length-dependent manner and to induce the formation of inclusions. Using rCGG oligoribonucleotide capture from brain lysates followed by mass spectrometry, an rCGG-repeatbinding protein (rCGGBP) has been identified. The interaction with rCGG repeat is conserved between mammals and Drosophila and is sequence specific. Furthermore, overexpression of rCGGBP in Drosophila suppresses rCGG-mediated neurodegeneration in a dose-dependent manner. These data support the disease mechanism of the fragile X premutation rCGG repeat sequestering specific rCGGBPs, leading to neuronal cell death.

DM1 and DM2 are primarily muscle diseases but also involve CNS pathology. The disease-causing potential of these mutant RNAs probably derives from the length of the repeat sequences and their interaction with RNA-binding proteins of the muscleblind-like (MBNL) family. MBNL proteins bind to expanded CUG and CCUG repeats, and the mass of repeat expansion RNA in DM cells is sufficient to sequester MBNL proteins and interfere with their function as regulators of alternative splicing. Several neuronal transcripts have been identified that show misregulated splicing in DM1, including tau, amyloid precursor protein, and NMDA NR1 receptor, but their connection with the CNS symptoms remains uncertain (Jiang et al., 2004). As evidence for such a protein sequestration mechanism, disruption of Muscleblind 1 in mice induces abnormalities of alternative splicing that are strikingly similar to those observed in human DM1 skeletal muscle (Kanadia et al., 2003). An interesting feature of RNAs with highly expanded CUG or CCUG repeats is that they are retained in nuclear foci. In cortical neurons and striated muscle, muscleblind proteins are recruited into the nuclear foci and depleted elsewhere from the nucleoplasm.

Similarly, other neurodegenerative conditions caused by nontranslated repeat expansions might involve pathogenic RNA mechanisms. These include spinocerebellar ataxias (SCA) type 8, 10, and 12 and Huntington's disease-like 2 (Ranum and Day, 2004). Particularly noticeable is SCA8, which is linked to a CUG repeat expansion in an untranslated transcript that induces progressive neurodegeneration in Drosophila (Mutsuddi et al., 2004). Because untranslated triplet repeats can cause neurodegeneration, a question that comes immediately to mind is whether RNA-based mechanisms also play a role in diseases caused by translated CAG repeats, which are all neurodegenera- tive, the best example being Huntington's disease. A strong argument against such a possibility is that polyglutamine sequences encoded by CAG or CAA repeats cause indistinguishable phenotypes in Drosophila and untranslated CAG repeats do not cause a phenotype in this system (McLeod et al., 2005). However SCA12 is caused by a CAG expansion in the $5^{\prime}$-UTR of the PPP2R2B gene and is phenotypically very similar to other SCAs caused by translated CAG repeats (Holmes et al., 1999). Expansions in CAG repeat disorders are more modest that in FXTAS and DM1, but they could potentially sequester RNA-binding proteins that may play a modulating role.

The potential implication of UTR-binding proteins in neurodegeneration is not limited to diseases caused by repeat expansions. For instance, overexpression of the $3^{\prime}$-UTR of the light neurofilament subunit (NF-L) mRNA causes motor neuron disease in mice (Nie et al., 2002) and accumulation of ubiquitinated aggregates in degenerating motor neurons in culture (Lin et al., 2003). The cognate RNA-binding protein p190RhoGEF binds to the $3^{\prime}$-UTR of NF-L mRNA and stabilizes it (Canete-Soler et al., 2001). Neurofilaments are particularly relevant to ALS because they accumulate in the perikarya of dying motor neurons in sporadic as well as familial forms of ALS caused by missense mutations in the superoxide dismutase 1 (SOD1) gene (Bruijn et al., 2004). In G93A mutant SOD1 transgenic mice, which develop a motor neuron disease phenotype reminiscent of the human disease, aggregates of NF-L, p190RhoGEF, and mutant SOD1 are very prominent in the vacuolated neuropil in the vicinity of degenerating motor neurons. Aggregates of NF-L are potentially neurotoxic in that they have synergistic effects on the aggregation of mutant, but not wild-type, SOD1 (Lin et al., 2004). Interestingly, similar aggregates of p190RhoGEF and NF-L occur in neurites surrounding degenerating motor neurons of mice expressing untranslated NF-L RNA in the 3'-UTR of a green fluorescent protein reporter transgene, suggesting that the aggregates themselves may be a triggering event in the pathogenesis of motor neuron degeneration (Lin et al., 2005). The findings support a model whereby interactions of $\mathrm{p} 190 \mathrm{RhoGEF}$ and NF-L protein lead to aggregation of NF-L protein with neurotoxic effects on motor neurons in motor neuron disease. The participation of p190RhoGEF in the pathogenesis of motor neuron degeneration is believed to derive from its prospective role in regulating steadystate levels of NF-L expression. p190RhoGEF binds and stabilizes NF-L mRNA and also interacts with unassembled NF-L protein and links stabilization of NF-L mRNA with the disposition of NF-L subunits in the cell. Interactions of p190RhoGEF lead to coaggregation with unassembled NF-L protein and downregulation of NF-L mRNA. However, p190RhoGEF does not interact with assembled NF-L subunits so that assembly of NF-L prevents the interactions of p190RhoGEF and NF-L protein that downregulate NF-L mRNA. Alteration of this regulatory pathway may lead to excessive aggregation of NF-L and p190RhoGEF with neurotoxic effects on motor neurons and could account for the selective loss of NF-L mRNA that occurs in motor neurons of mice bearing mutant SOD1 transgenes and of patients with familial and sporadic ALS.

\section{Alternative splicing in neurodegeneration: mechanisms and RNA-based therapies}

Disruption of the exquisite functional diversity provided by isoforms arising from a single gene by alternative pre-mRNA splicing can also have neurotoxic consequences. The selection of specific $5^{\prime}$ and $3^{\prime}$ splice sites in alternative splicing events involves exonic and intronic splicing silencer and enhancer elements and 
splicing factors binding to these elements, especially members of the serine-arginine (SR)-rich protein family. Alternative splicing can be disrupted through stress from a primary condition modifying the activity of splicing factors or by mutations affecting splicing elements.

The first example comes from ALS in which, in addition to neurofilaments, another neuronal intermediate filament protein, peripherin, forms intraneuronal proteinaceous aggregates and is overexpressed in motor neurons. Three splice variants of peripherin have been identified in the mouse: Per 58, which is encoded by all nine exons of the peripherin gene; Per 56, generated by the use of a cryptic acceptor site at the beginning of exon 9 that results in a frame shift and replacement of the C-terminal 21 amino acids of Per 58 with a unique eight amino acid sequence; and Per 61, which is generated by the in-frame retention of intron 4 leading to a 32 amino acid insertion within a domain of peripherin crucial for intermediate filament assembly. Per 58 and Per 56 appear to be normal isoforms in motor neurons of wild-type mice, whereas Per 61 is not. However, Per 61 is expressed in motor neurons of mutant SOD1 transgenic mice, indicating that mutant SOD1 induces differential splicing of the peripherin pre-mRNA (Robertson et al., 2003). Per 61 is toxic to primary motor neurons in culture and also induces peripherin and neurofilament aggregate formation. It is likely that alternative splicing of the peripherin pre-mRNA also occurs in humans; indeed, several expressed sequence tags representing alternatively spliced variants of peripherin can be found in the National Center for Biotechnology Information database. Finding ALS-specific splicing events involving peripherin, and perhaps other genes, will open the way to identifying new pathogenic mechanisms in ALS.

The mechanistic importance of impaired alternative splicing in neurodegeneration has been ascertained by the discovery of mutations in the MAPT gene, encoding the microtubuleassociated protein, tau, in frontotemporal dementia with parkinsonism linked to chromosome 17 (FTDP-17) (Hutton et al., 1998). FTDP-17 is part of the group of dementias characterized by intraneuronal deposition of tau and collectively referred to as tauopathies that include Alzheimer's disease and also DM1. Exon 10 (E10) of the MAPT gene encodes the second of four imperfect microtubule-binding repeats in the C-terminal half of the tau protein. Tau pre-mRNA is alternatively spliced to produce $\mathrm{E} 10^{+}$ and $\mathrm{E} 10^{-}$tau isoforms that are expressed in approximately equal amounts in adult human brain (Avila et al., 2004). Sixteen missense, silent, deletion, and intronic FTDP-17 mutations affect 7 of 10 splicing regulatory sequences, which include both tau E10 splice sites as well as several splicing enhancer and silencer elements in E10 and in the flanking introns (D'Souza and Schellenberg, 2002). E10 retention in FTDP-17 results in a twofold to sixfold excess of $\mathrm{E} 10^{+}$tau over E10 ${ }^{-}$tau (Hutton et al., 1998; Connell et al., 2005). The identity and function of trans-factors that interact with cis-regulatory sequences offer clues to normal and aberrant tau E10 splicing. Using in vitro binding assays and in vivo knock-down and overexpression assays, the SR splicing factor splicing factor 2 (SF2)/alternate splicing factor (ASF) and the SR-like splicing factor Tra $2 \beta$ that bind the normal E10 polypurine enhancer (PPE) element were identified (Jiang et al., 2003). FTDP-17 mutations $\Delta 280 \mathrm{~K}$ and N279K disrupt the PPE and inhibit or enhance E10 splicing, respectively, through altered associations with SF2/ASF and Tra $2 \beta$. Mutation $\Delta 280 \mathrm{~K}$ abolishes all in vitro interactions, whereas mutation $\mathrm{N} 279 \mathrm{~K}$ increases Tra $2 \beta$ binding. Interestingly, overexpression only of SF2/ASF restores splicing in $\Delta 280 \mathrm{~K}$. The general involvement of other SR and non-SR splicing factors is currently being studied using knock-

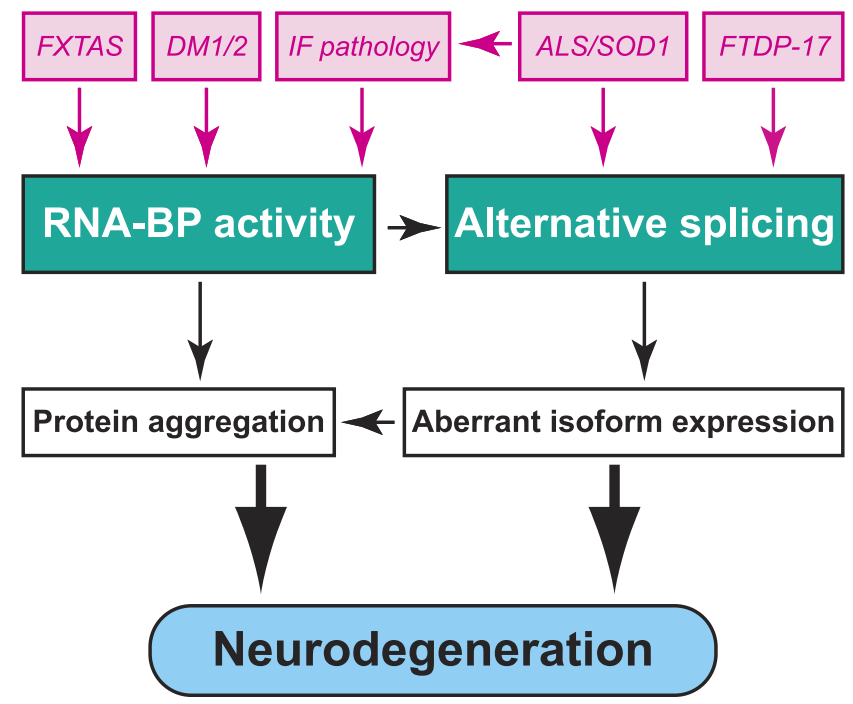

Figure 1. Diagram summarizing the involvement of RNA-mediated mechanisms in the neurodegenerative process in the nontranslated triplet repeat expansion diseases, FXTAS and DM1 and DM2, FTDP-17, and disorders with intermediate filament (IF) pathology. The latter refer to motor neuron diseases with accumulations of neurofilaments or peripherin, which include sporadic and SOD1-linked amyotrophic lateral sclerosis (ALS/SOD1). In this diagram, aggregation is used sensu lato, encompassing microscopically visible inclusions as well as smaller oligomers.

down assays. Transgenic mice that express normal and mutant human tau genes have been generated to determine how FTDP-17 splicing mutations alter the spatiotemporal expression pattern of E10 in the developing CNS. The expression of critical splicing factors in relation to E10 levels in different brain regions will be instrumental in understanding how subtle changes in E10 expression contribute to the clinical variability seen not only in FTDP-17 but also in other related dementias.

From a therapeutic perspective, correcting isoform imbalance, be it tau or another transcript, would require ideally direct intervention at the RNA level, hence maintaining endogenous transcriptional control. Reduction of E10 inclusion has been achieved by using oligonucleotides binding to E10 splice junctions in PC12 cells that express predominantly E10 ${ }^{+}$tau (Kalbfuss et al., 2001). Another, more versatile strategy is to reprogram tau mRNA using spliceosome-mediated RNA trans-splicing, or SMaRT (Puttaraju et al., 1999). SMaRT creates a chimeric mRNA through a trans-splicing reaction mediated by the spliceosome between the $5^{\prime}$ splice site of an endogenous target pre-mRNA and the $3^{\prime}$ splice site of an exogenously delivered pre-trans-splicing RNA molecule. Tau pre-mRNA is a suitable target for SMaRT at the level of E10 splicing, and the 3' end of the tau message can be replaced by the $3^{\prime}$ end of a pre-trans-splicing molecule containing human tau exons 10-13 and a targeting domain binding to intron 9. Furthermore, cis-splicing exclusion of E10 can be bypassed by trans-splicing, and conversion of E10 ${ }^{-}$tau RNA into $\mathrm{E}{ }^{+}$RNA can be achieved with an efficiency of $\sim 30 \%$ (Rodriguez-Martin et al., 2005). Thus, trans-splicing strategies can be used to replace efficiently alternatively spliced exons and suggest a potential therapeutic application of SMaRT not only for tauopathies but also for other disorders linked to impairment of alternative splicing.

\section{Concluding remarks}

The above examples illustrated the implication of RNA-based neurotoxic mechanisms in a number of neurodegenerative conditions. The putative mechanisms involved are summarized in 
Figure 1. Disruption of alternative splicing regulation, resulting directly from either mutations affecting splicing regulatory elements or abnormal activity of RNA-binding proteins, can upset a tightly regulated tissue and developmentally regulated expression program leading to imbalance or inappropriate expression of isoforms of key proteins. Novel trans-dominant mechanisms offer a new perspective on the role of RNA in neurodegenerative processes. Surprisingly, both aspects of RNA-based mechanisms are linked to protein misfolding and aggregation, a hallmark of neurodegeneration. The realization that aberrant RNA processing is a mediator of at least some neurodegenerative processes point to specific RNAs as targets for novel therapeutic interventions.

\section{References}

Avila J, Lucas JJ, Perez M, Hernandez F (2004) Role of tau protein in both physiological and pathological conditions. Physiol Rev 84:361-384.

Bruijn LI, Miller TM, Cleveland DW (2004) Unraveling the mechanisms involved in motor neuron degeneration in ALS. Annu Rev Neurosci 27:723-749.

Canete-Soler R, Wu J, Zhai J, Shamim M, Schlaepfer WW (2001) p190RhoGEF binds to a destabilizing element in the $3^{\prime}$ untranslated region of light neurofilament subunit mRNA and alters the stability of the transcript. J Biol Chem 276:32046-32050.

Connell JW, Rodriguez-Martin T, Gibb GM, Khan NM, Grierson AJ, Hanger DP, Revesz T, Anderton BH, Gallo J-M (2005) Quantitative analysis of tau isoform transcripts in sporadic tauopathies. Brain Res Mol Brain Res 137:104-109.

D’Souza I, Schellenberg GD (2002) Tau exon 10 expression involves a bipartite intron 10 regulatory sequence and weak $5^{\prime}$ and $3^{\prime}$ splice sites. J Biol Chem 277:26587-26599.

Frugier T, Nicole S, Cifuentes-Diaz C, Melki J (2002) The molecular bases of spinal muscular atrophy. Curr Opin Genet Dev 12:294-298.

Gubitz AK, Feng W, Dreyfuss G (2004) The SMN complex. Exp Cell Res 296:51-56.

Hagerman PJ, Hagerman RJ (2004) The fragile-X premutation: a maturing perspective. Am J Hum Genet 74:805-816.

Holmes SE, O’Hearn EE, McInnis MG, Gorelick-Feldman DA, Kleiderlein JJ, Callahan C, Kwak NG, Ingersoll-Ashworth RG, Sherr M, Sumner AJ, Sharp AH, Ananth U, Seltzer WK, Boss MA, Vieria-Saecker AM, Epplen JT, Riess O, Ross CA, Margolis RL (1999) Expansion of a novel CAG trinucleotide repeat in the $5^{\prime}$ region of $P P P 2 R 2 B$ is associated with SCA12. Nat Genet 23:391-392.

Hutton M, Lendon CL, Rizzu P, Baker M, Froelich S, Houlden HH, Pickering Brown S, Chakraverty S, Isaacs A, Grover A, Hackett J, Adamson J, Lincoln S, Dickson D, Davies P, Petersen RC, Stevena M, De Graaff E, Wauters E, et al. (1998) Association of missense and 5'-splice-site mutations in tau with the inherited dementia FTDP-17. Nature 393:702-704.

Jiang H, Mankodi A, Swanson MS, Moxley RT, Thornton CA (2004) Myotonic dystrophy type 1 is associated with nuclear foci of mutant RNA, sequestration of muscleblind proteins and deregulated alternative splicing in neurons. Hum Mol Genet 13:3079-3088.

Jiang Z, Tang H, Havlioglu N, Zhang X, Stamm S, Yan R, Wu JY (2003) Mutations in tau gene exon 10 associated with FTDP-17 alter the activity of an exonic splicing enhancer to interact with $\operatorname{Tra} 2 \beta$. J Biol Chem 278:18997-19007.

Jin P, Zarnescu DC, Zhang F, Pearson CE, Lucchesi JC, Moses K, Warren ST
(2003) RNA-mediated neurodegeneration caused by the fragile X premutation rCGG repeats in Drosophila. Neuron 39:739-747.

Kalbfuss B, Mabon SA, Misteli T (2001) Correction of alternative splicing of tau in frontotemporal dementia and parkinsonism linked to chromosome 17. J Biol Chem 276:42986-42993.

Kanadia RN, Johnstone KA, Mankodi A, Lungu C, Thornton CA, Esson D, Timmers AM, Hauswirth WW, Swanson MS (2003) A muscleblind knockout model for myotonic dystrophy. Science 302:1978-1980.

Kosik KS, Krichevsky AM (2005) The elegance of the microRNAs: a neuronal perspective. Neuron 47:779-782.

Lin H, Zhai J, Nie Z, Wu J, Meinkoth JL, Schlaepfer WW, Canete-Soler R (2003) Neurofilament RNA causes neurodegeneration with accumulation of ubiquitinated aggregates in cultured motor neurons. J Neuropathol Exp Neurol 62:936-950.

Lin H, Zhai J, Canete-Soler R, Schlaepfer WW (2004) 3' untranslated region in a light neurofilament (NF-L) mRNA triggers aggregation of NF-L and mutant superoxide dismutase 1 proteins in neuronal cells. J Neurosci 24:2716-2726.

Lin H, Zhai J, Schlaepfer WW (2005) RNA-binding protein is involved in aggregation of light neurofilament (NF-L) protein and is implicated in the pathogenesis of motor neuron degeneration. Hum Mol Genet, in press.

McLeod CJ, O'Keefe LV, Richards RI (2005) The pathogenic agent in Drosophila models of "polyglutamine" diseases. Hum Mol Genet 14:1041-1048.

Mutsuddi M, Marshall CM, Benzow KA, Koob MD, Rebay I (2004) The spinocerebellar ataxia 8 noncoding RNA causes neurodegeneration and associates with staufen in Drosophila. Curr Biol 14:302-308.

Nie Z, Wu J, Zhai J, Lin H, Ge W, Schlaepfer WW, Canete-Soler R (2002) Untranslated element in neurofilament mRNA has neuropathic effect on motor neurons of transgenic mice. J Neurosci 22:7662-7670.

Puttaraju M, Jamison SF, Mansfield SG, Garcia-Blanco MA, Mitchell LG (1999) Spliceosome-mediated RNA trans-splicing as a tool for gene therapy. Nat Biotechnol 17:246-252.

Ranum LP, Day JW (2004) Pathogenic RNA repeats: an expanding role in genetic disease. Trends Genet 20:506-512.

Robertson J, Doroudchi MM, Nguyen MD, Durham HD, Strong MJ, Shaw G, Julien JP, Mushynski WE (2003) A neurotoxic peripherin splice variant in a mouse model of ALS. J Cell Biol 160:939-949.

Rodriguez-Martin T, Garcia-Blanco MA, Mansfield SG, Grover A, Hutton M, Yu Q, Zhou J, Anderton BH, Gallo J-M (2005) Reprogramming of tau alternative splicing by spliceosome-mediated RNA trans-splicing: implications for tauopathies. Proc Natl Acad Sci USA 102:15659-15664.

Rossoll W, Jablonka S, Andreassi C, Kroning AK, Karle K, Monani UR, Sendtner M (2003) Smn, the spinal muscular atrophy-determining gene product, modulates axon growth and localization of $\beta$-actin mRNA in growth cones of motoneurons. J Cell Biol 163:801-812.

Tassone F, Hagerman RJ, Taylor AK, Gane LW, Godfrey TE, Hagerman PJ (2000) Elevated levels of FMR1 mRNA in carrier males: a new mechanism of involvement in the fragile-X syndrome. Am J Hum Genet 66:6-15.

Ule J, Ule A, Spencer J, Williams A, Hu JS, Cline M, Wang H, Clark T, Fraser C, Ruggiu M, Zeeberg BR, Kane D, Weinstein JN, Blume J, Darnell RB (2005) Nova regulates brain-specific splicing to shape the synapse. Nat Genet 37:844-852.

Willemsen R, Hoogeveen-Westerveld M, Reis S, Holstege J, Severijnen LA, Nieuwenhuizen IM, Schrier M, Van Unen L, Tassone F, Hoogeveen AT, Hagerman PJ, Mientjes EJ, Oostra BA (2003) The FMR1 CGG repeat mouse displays ubiquitin-positive intranuclear neuronal inclusions; implications for the cerebellar tremor/ataxia syndrome. Hum Mol Genet 12:949-959. 